\title{
Amyloid associated with elastin-staining laminar aggregates in the lungs of patients diagnosed with acute respiratory distress syndrome
} Kang Fan*1,3 and William A Nagle 2,4,5

Address: ${ }^{1}$ Pathology and Laboratory Medicine Service, Central Arkansas Veterans Healthcare System, Little Rock, AR 72205, USA, ${ }^{2}$ Nuclear Medicine Service, Central Arkansas Veterans Healthcare System, Little Rock, AR 72205, USA, ${ }^{3}$ Department of Pathology, University of Arkansas for Medical Sciences, Little Rock, AR 72205, USA, ${ }^{4}$ Department of Radiology, University of Arkansas for Medical Sciences, Little Rock, AR 72205, USA and ${ }^{5}$ Department of Physiology and Biophysics, University of Arkansas for Medical Sciences, Little Rock, AR 72205, USA

E-mail: Kang Fan* - fankang@uams.edu; William A Nagle - naglewilliama@uams.edu

${ }^{*}$ Corresponding author

Published: II October 2002

BMC Pulmonary Medicine 2002, 2:5
Received: 30 April 2002

Accepted: II October 2002

This article is available from: http://www.biomedcentral.com//47I-2466/2/5

(C) 2002 Fan and Nagle; licensee BioMed Central Ltd. This article is published in Open Access: verbatim copying and redistribution of this article are permitted in all media for any purpose, provided this notice is preserved along with the article's original URL.

\begin{abstract}
Background: The heterogeneity of conditions underlying respiratory distress, whether classified clinically as acute lung injury (ALI) or the more severe acute respiratory distress syndrome (ARDS), has hampered efforts to identify and more successfully treat these patients. Examination of postmortem lungs among cases clinically diagnosed as ARDS identified a cohort that showed a consistent morphology at the light and electron microscope levels, and featured pathognomonic structures which we termed elastin-staining laminar structures (ELS).

Methods: Postmortem tissues were stained using the Verhoeff-Van Gieson procedure for elastic fibers, and with Congo red for examination under a polarizing microscope. Similar samples were examined by transmission EM.

Results: The pathognomonic ELS presented as ordered molecular aggregates when stained using the Verhoeff-van Gieson technique for elastic fibers. In several postmortem lungs, the ELS also displayed apple-green birefringence after staining with Congo red, suggesting the presence of amyloid. Remarkably, most of the postmortem lungs with ELS exhibited no significant acute inflammatory cellular response such as neutrophilic reaction, and little evidence of widespread edema except for focal intra-alveolar hemorrhage.

Conclusions: Postmortem lungs that exhibit the ELS constitute a morphologically-identifiable subgroup of ARDS cases. The ordered nature of the ELS, as indicated by both elastin and amyloid stains, together with little morphological evidence of inflammation or edema, suggests that this cohort of ARDS may represent another form of conformational disease. If this hypothesis is confirmed, it will require a new approach in the diagnosis and treatment of patients who exhibit this form of acute lung injury.
\end{abstract}

\section{Background}

Respiratory distress marked by acute lung injury (ALI), or its more severe form, acute respiratory distress syndrome (ARDS), is a significant health problem worldwide. In the 
United States, the annual incidence of ALI and ARDS together is thought to be about 75 per 100,000 population [1], with the incidence of ARDS alone probably exceeding 100,000 cases per year. The mortality rates reported for ARDS are highly variable, ranging from as low as 10 to as high as 90 percent [2], owing at least in part to the "lack of a uniform definition and the heterogeneity of the causes and clinical manifestations" of the syndrome [1]. Such uncertainty has hampered efforts to identify patients at risk for developing ARDS, and subsequently, for the development of more successful therapeutic interventions. It has been suggested [3] that "ARDS" probably encompasses several distinct states, each of which results from a different initiating event, but all of which eventually are expressed as the common respiratory distress sequelae.

We have identified a subset of postmortem ARDS cases based on consistent morphological features at both the gross and microscopic levels. A defining feature of this cohort is the presence, within the alveoli and terminal air spaces, or adherent to the alveolar linings, of ordered molecular aggregates which we termed "elastin-staining laminar structures" (ELS), because they stain positively using the Verhoeff-Van Gieson technique for elastic fibers. In an earlier report [4], we described preliminary experiments to characterize the ELS in vitro. Because isolated ELS were found to be protease-resistant, fibrillar aggregates in vitro, we asked whether they might contain amyloid in situ. Here, we describe ultrastructural features of the ELS in situ, and in addition, show that the ELS from several different postmortem cases exhibit green birefringence after staining with Congo red, a characteristic of amyloid. Thus, we suggest that cases of respiratory distress exhibiting the ELS represent a morphologically definable subclass of ARDS, and hypothesize that the pulmonary accumulation of ELS might represent a new type of conformational disease.

\section{Methods}

\section{Selection and description of cases}

All materials used were obtained from autopsies performed at the Central Arkansas Veterans Healthcare System (CAVHS), within 8 hours of death, using standard postmortem examination procedures. All autopsies were done with legally signed permits, and all privacy acts regulations were followed. The study was entirely retrospective; no sequential or kinetic measurements were made. All patients with respiratory distress had received standard hospital respiratory care, which included mechanical-assisted ventilation where clinically appropriate.

We first screened for the presence of the ELS by examining sections from each lobe of the post-mortem lungs as described below. In this study, the ELS were found in 30 different cases from about 100 total cases screened: all 30 ELS cases either showed acute lung injury, or satisfied the clinical criteria for ARDS (using the criteria established by the 1992 American-European Consensus Conference on ARDS [2], i.e., $\mathrm{PaO}_{2}: \mathrm{FiO}_{2}<200 \mathrm{~mm} \mathrm{Hg}$, and bilateral infiltrates on frontal chest radiographs), but otherwise constituted a heterogeneous group with respect to underlying clinical diseases.

Cases that did not demonstrate the ELS included, for example, various types of pneumonia and interstitial pneumonitis, ranging from bronchopneumonia, lung abscess, and bronchiolitis obliterans organizing pneumonia (BOOP), and lungs with idiopathic or established pulmonary interstitial fibroses when these cases did not present clinical evidence of ARDS.

\section{Specimen preparation, specific staining, and microscopy}

The formalin fixed postmortem lung tissues were processed using routine procedures, initially stained with $\mathrm{H} \& \mathrm{E}$, and then examined microscopically. Those sections that showed hyaline membrane-like components were then stained for elastic fibers, using the Verhoeff-Van Gieson technique [5]. Lung sections from all 30 ELS cases described above were then stained with Congo red, using the alcoholic, high-salt procedure of Puchtler et al [6], as described by Lillie and Fullmer [5], and examined under polarized light using a Leitz microscope. Selected tissues also were processed for EM, in order to examine the ELS at the ultrastructural level. Routine transmitting EM was performed as follows. The tissue was initially fixed in $4 \%$ gluteraldehyde and processed with the standard Osmium tetroxide, ethanol gradient, propylene oxide and then embedded in Epon resin before sectioning. The grids were stained with uranyl acetate and lead, and then examined using a Joel model JEM-110 transmitting electron microscope.

\section{Results}

Postmortem lungs from the ELS cohort of ARDS cases display a relatively well preserved anatomical lung structure. Tissue sections stained by $\mathrm{H} \& \mathrm{E}$ and viewed with the light microscope show hyaline membrane-like structures that often adhere to the alveolar septi, and occasionally are seen in the terminal air spaces, similar to those originally reported by Ashbaugh et al [7]. However, after staining by the Verhoeff's Van Gieson elastin method, the pathognomonic ELS are demonstrated: they present mostly as ordered, laminar structures of varying thickness, separated by non-staining proteinaceous inserts (Figure 1). Notably, the structurally-definable ELS corresponded anatomically to portions of what appeared to be hyaline-like material in the H\&E sections. In general, postmortem lungs showing the ELS: 1) displayed no significant acute inflammatory cellular exudates such as neutrophils, that were previously described as being an essential component for ARDS [8]; and 2) showed no evidence of preexisting inter- 


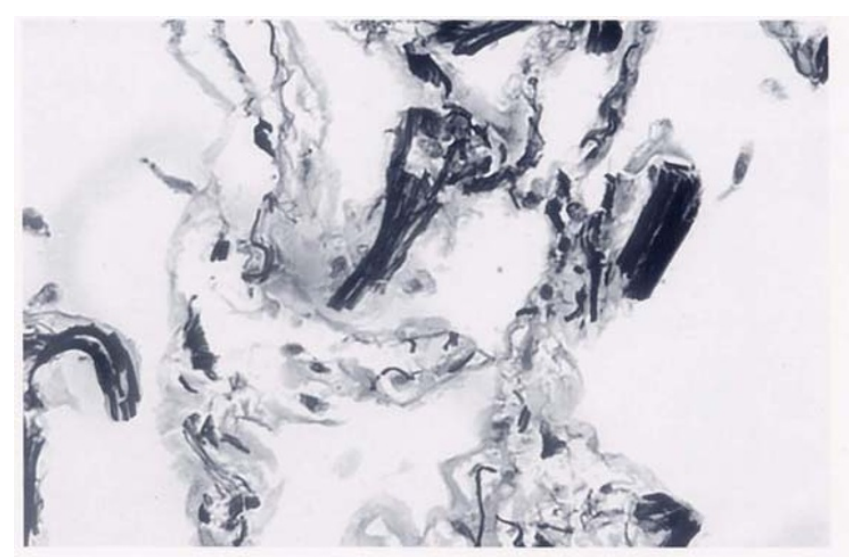

Figure I

Representative photomicrograph of a Verhoeff-Van Gieson elastin stained postmortem lung from a patient who died after acute respiratory distress. Elastin-staining laminar structures (ELS) are prominently featured. Some of the ELS are partially attached to the alveolar septa. Inflammatory cellular exudates are essentially absent in the alveoli of terminal respiratory spaces. $(200 \times)$

stitual fibrosis or of fibroblastic proliferation. Moreover, about one-half of the ELS cases exhibited no evidence of significant edema, except for focal intra-alveolar hemorrhage.

EM examination of postmortem lung tissue containing the ELS reveals distinct abnormalities, and dramatically illustrates the appearance ELS in situ (Figure 2). Again, the ELS exhibit a distinctly laminar and ordered structure, with a fibril-like appearance at the edges. Little evidence is seen of the amorphous component of elastic fibers, which in normal, mature elastic tissue can constitute up to 90 percent of the fiber content [9]. Thus, while morphologically intact constitutional alveolar septum collagen fibrils are seen, without evidence of alternation of the banding periodicity, morphologically normal septum constitutional elastin fibers are not demonstrable. Several ELS showing rigid, seemingly broken, ends are noted within the alveolar septum, and bordered by alveolar air spaces and the epithelium cytoplasmic membrane. However, capillaries within the septum appear morphologically normal, and RBCs are still confined within the capillary lumen (Figure 2).

Three cases (among the ELS cohort of 30 cases) displayed ELS-associated apple-green birefringence after Congo red staining; Figure 3 shows an example, taken from the same case (case \#2 below) as the tissue used for the EM photomicrograph (Figure 2). The three cases comprised a heterogeneous group, including septic shock secondary to peritonitis (acute diverticulitis with perforation); malig-

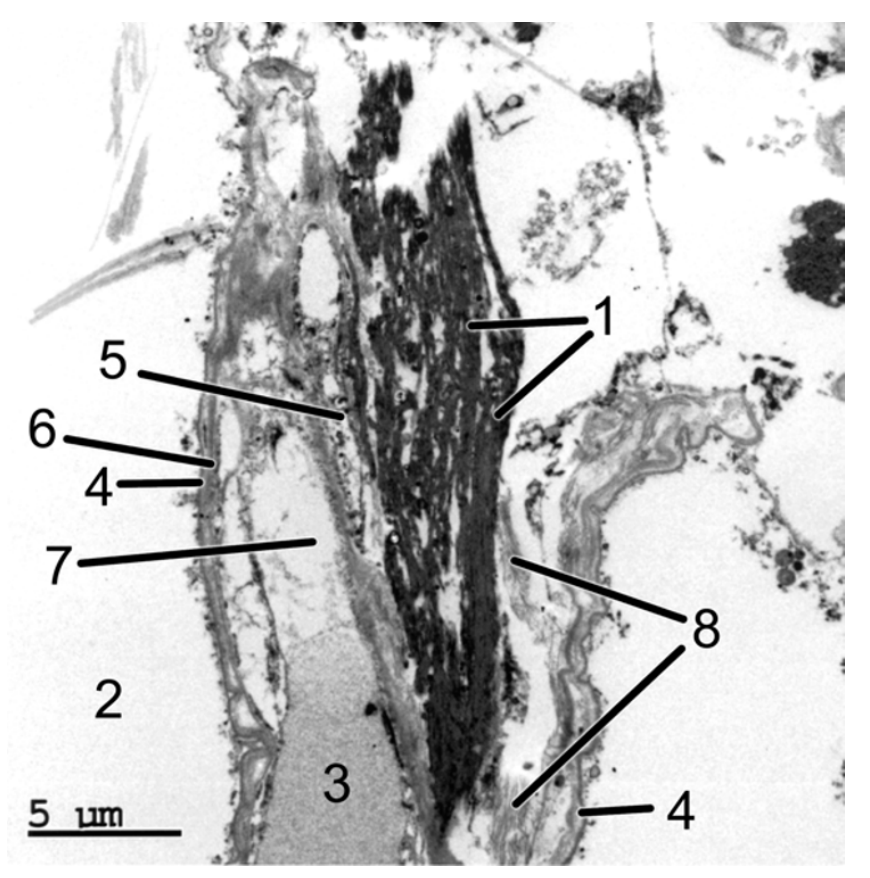

Figure 2

EM morphology of the postmortem ELS lung (the same case as shown in Figure I). The ELS are seen within the alveolar septum bordered by the basement membrane of the alveolar space on each side. The EM image dramatically demonstrates the in situ nature of the ELS: I = ELS; 2 = alveolar space; $3=$ erythrocyte; 4 = basement membrane; 5 = endothelial cell nucleus; 6 = endothelial cell plasma membrane; $7=$ capillary plasma; 8 = collagen fibers. $(50,000 \times)$

nant trauma (lymphoma); and trauma associated with major surgery (acute cerebral hemorrhage). In these three cases, green birefringence after Congo red staining was not observed in other tissues, with the exception of an occasional positive muscular arterial wall (case \#1), and the heart and kidney (case \#2).

\section{Discussion}

Apple green birefringence of amyloid deposits stained with Congo red and examined using polarized light "remains the gold standard" [10] for the diagnosis of amyloid fibrils in vivo, and is thought to be due to two main factors [11]: 1) a parallel arrangement of dye molecules in the sample, and 2) a thickness of the stained sample that will result in extinction of the red component of the light with appearance of the apple green color. Thus, the appearance of green birefringence with Congo red confirms the presence of ordered, amyloid-like structures in these cases of ARDS. The reason why only three of the 30 ELS cases exhibited green birefringence with Congo red cannot be explained at this time, because the molecular composition of the ELS remains unknown. We see no apparent structural differences in those ELS that are Con- 


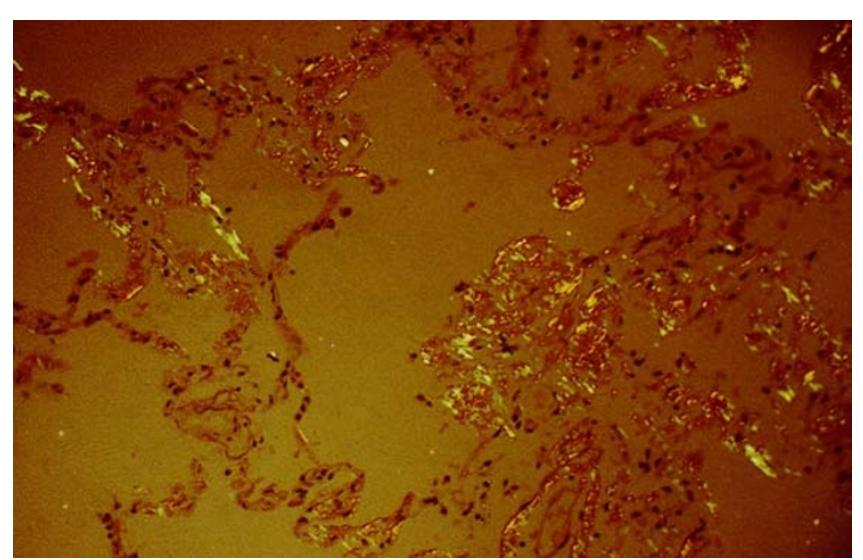

\section{Figure 3}

Photomicrograph of Congo red-stained lung tissue of the same case as shown in Figure I, viewed under cross-polarized light. Apple green birefringence is amply demonstrated in the ELS structures. (100x).

go red birefringence-positive and those that are birefringence-negative, at either the light or electron microscope levels. Studies by others suggest that factors in addition to stained sample thickness may affect Congo red birefringence, including morphological differences in the amyloid fibrils themselves $[12,13]$, and the presence of nonfibril components such as additional polypeptides and carbohydrate [14]. The three cases that showed birefringence with Congo red in this study were also examined and found positive for amyloid using two other procedures: 1) fluorescence with thioflavin $\mathrm{T}$, and 2) reaction with antiserum to the amyloid P component (AP) glycoprotein (data not shown).

Currently about 20 different proteins have been associated with amyloid deposits in vivo in humans $[14,15]$. Several earlier reports noted an association of structures that both stained positive with the Verhoeff-van Gieson procedure, and exhibited characteristics of amyloid of undetermined origin in pathological conditions in the cornea $[16,17]$ and skin $[18]$. The suggestion was made that "the amyloid-like filaments actually represented microfibrils of elastic tissue"[18]; however, that hypothesis has not been confirmed to our knowledge. Likewise, we are unaware of any prior reports of the appearance of ordered molecular aggregates that stain positively for both amyloid and elastin in cases of acute lung injury.

Altered or aberrant protein folding leading to aggregation has been suggested as the molecular basis of an increasing number of human diseases [19-21], now referred to as "conformational diseases" [22] or "proteopathies" [23], in order to distinguish them by pathological criteria from inflammatory, degenerative, or neoplastic diseases. In the present study, the amyloid tests and staining with the Verhoeff-Van Gieson technique both showed the ELS to be ordered molecular aggregates. The histochemical evidence further suggests that normal elastic fibers underwent substantial structural changes, and became part of the observed ELS seen in these postmortem ARDS lungs. The involvement of elastic fibers in structural changes in ARDS lungs is not unprecedented. For example, fragments of elastin have been detected in the levage of ARDS patients [3], and Negri et al [24] showed evidence of significant elastic fiber remodeling in fibroproliferative ARDS lungs.

Elastic fiber restructuring is consistent with two clinical manifestations of ARDS observed in the ELS cohort of individuals, namely severe hypoxemia $\left(\mathrm{PaO}_{2}: \mathrm{FiO}_{2}<200\right.$ $\mathrm{mm} \mathrm{Hg}$ ), and rapid progression of clinical symptoms in spite of little evidence of acute or specific chronic inflammation, or widespread edema. First, elastic fibers constitute an important component of the alveolar interstitium, where they are thought to be responsible for the ability of the lung tissue to stretch and recoil [25]. The EM morphology suggests that: (1) the free ELS in the alveolar air spaces could result from mechanical disruption of the septum-lining basement membrane by the rigid ELS, whose ends appear sharp and broken; and (2) the structural changes observed, evidently associated with formation of the ELS aggregates, constitute the major pathological changes in that lung tissue. Disruption of the normal structure of the elastic fibers (such as their apparent fragmentation and aggregation into the ELS as seen here) would be expected to result in severe mechanical malfunction of the lung (decreased compliance) together with a reduced capability for normal gas exchange. Secondly, the ELS were only observed in those cases of clinical ARDS characterized by a rapidly developing respiratory failure; this observation suggests an acute, episodic form of conformational disease, similar to that described for severe episodic thrombosis initiated by fever [26]. As yet, the stimuli responsible for ELS formation in ARDS have not been identified.

In most lungs that show the ELS, we do not observe either the presence of substantial numbers of inflammatory cells or accumulations of fibrotic materials. While we can not rule out a role for soluble mediators in the structural changes observed, large numbers of inflammatory cells or fibroblasts do not appear necessary for ELS formation. Nor do these factors appear sufficient, because lungs that show any or all of these markers often do not exhibit the ELS. Thus, the ELS always are associated with respiratory failure; however, respiratory failure is not a predictor of the presence of the ELS. We propose that the ELS define a structure-based subgroup of ARDS cases, that may share a common etiology. If the conformational disease hypothesis for the ELS cohort of ARDS cases is confirmed, subse- 
quent efforts may then be directed to develop more rational diagnostic and therapeutic procedures for these patients. A recent report summarizes the prospects for therapeutic intervention in cases of conformational disease [27].

\section{Competing interests}

None declared

\section{Authors' contributions}

K.F. made the initial observations of the ELS, and contributed and interpreted the clinical material presented here. W.A.N. provided the background for the conformational disease hypothesis, and drafted the manuscript.

\section{Abbreviations}

ALI: acute lung injury; ARDS: acute respiratory distress syndrome; ELS: elastin-staining laminar structures; EM: electron microscope

\section{Acknowledgements}

We thank Monica J. Crocker for carrying out the transmission EM procedures, and Donna Chapman for assistance with the figures.

\section{References}

I. Ware LB, Matthay MA: The acute respiratory distress syndrome. N Engl J Med 2000, 342:1334-1349

2. Bernard GR, Artigas A, Brigham KL, Carlet J, Falke K, Hudson L, et al: The American-European Consensus Conference on ARDS. Definitions, mechanisms, relevant outcomes, and clinical trial coordination. Am Journal Respir Crit Care Med 1994, I49:8I 8-824

3. Demling $\mathrm{RH}$ : The modern version of adult respiratory distress syndrome. Annual Review of Medicine 1995, 46:193-202

4. Helsel J, Yeh A, Fan K: Diagnostic and chemical properties of laminated elastin rich structures characteristic of adult respiratory distress syndrome (Stowell-Orbison Award-Winning Presentation, 1999 IAP Meeting, San Francisco, CA). Laboratory Investigation 79, 6a-6a. 1999

5. Lillie RD, Fullmer HM: Amyloid. In Histopathologic Technic and Practical Histochemistry New York: McGraw-Hill Book Company 1976, 663-666

6. Puchtler H, Sweat F, Levine M: On the binding of Congo red by amyloid. Journal of Histochemistry \& Cytochemistry 200I, 10:355-364

7. Ashbaugh DG, Bigelow DB, Petty TL, Levine BE: Acute respiratory distress in adults. Lancet 1967, 2:319-323

8. Weinacker AB, Vaszar LT: Acute respiratory distress syndrome: Physiology and new management strategies. Annual Review of Medicine 200I, 52:22I-237

9. Uitto J, Ryhanen LJ: Pathology of the elastic fibers. In Connective Tissue Disease (Edited by: Uitto J, Perejda AJ) New York: Marcel Dekker, Inc. 1987, 399-422

10. Pepys MB: Amyloidosis. In The Oxford Textbook of Medicine (Edited by: Weatherall DJ, Ledingham JGG, Warell DA) Oxford: Oxford University Press 1996, 1512-1524

II. Sunde M, Blake C: The structure of amyloid fibrils by electron microscopy and X-ray diffraction. Advances in Protein Chemistry 1997, 50:123-159

12. Goldfarb LG, Brown P, Haltia M, Ghiso J, Frangione B, Gajdusek DC: Synthetic peptides corresponding to different mutated regions of the amyloid gene in familial Creutzfeldt-Jakob disease show enhanced in vitro formation of morphologically different amyloid fibrils. Proc Natl Acad Sci USA 1993, 90:445I4454

13. Sunde M, Serpell LC, Bartlam M, Fraser PE, Pepys MB, Blake CC: Common core structure of amyloid fibrils by synchrotron $X$ ray diffraction. I Mol Biol 1997, 273:729-739

14. Picken MM: The changing concepts of amyloid. Archives of Pathology \& Laboratory Medicine 200 I, 1 25:38-43
15. Sipe JD, Cohen AS: Review: History of the amyloid fibril. Journal of Structural Biology 2000, 130:88-98

16. Dubord PJ, Rodrigues MM, Krachmer JH: Corneal elastosis in lattice corneal dystrophy: a clinicopathologic report. Ophthalmology 1981, 88:1239-1243

17. Pe'er J, Fine BS, Dixon A, Rothberg DS: Corneal elastosis within lattice dystrophy lesions. British Journal of Ophthalmology 1988, 72:183-188

18. Patterson JW, Wilkin JK, Schatzki PF: Nodular colloid degeneration: distinctive histochemical and ultrastructural features. Cutis 1985, 36:355-358

19. Thomas PJ, Qu B-H, Pedersen PL: Defective protein folding as a basis of human disease. Trends Biochem Sci 1995, 20:456-459

20. Dobson CM: Protein misfolding, evolution and disease. Trends Biochem Sci 1999, 24:329-332

21. Radford SE, Dobson CM: From computer simulations to human disease: emerging themes in protein folding. Cell 1999, 97:29|298

22. Carrell RW, Lomas DA: Conformational disease. Lancet 1997 , $350: 134-138$

23. Walker LC, LeVine H: The cerebral proteopathies. Neurobiology of Aging 2000, $21: 559-561$

24. Negri EM, Montes GS, Saldiva PH, Capelozzi VL: Architectural remodelling in acute and chronic interstitial lung disease: fibrosis or fibroelastosis? Histopathology 2000, 37:393-40I

25. Soskel NT, Sandberg LB: Pulmonary emphysema: From animal models to human disease. In Connective Tissue Disease (Edited by: Uitto J, Perejda A/) New York: Marcel Dekker, Inc. 1987, 423-453

26. Beauchamp NJ, Pike RN, Daly M, Butler L, Makris M, Dafforn TR, et al: Antithrombins Wibble and Wobble (T85M/K): archetypal conformational diseases with in vivo latent-transition, thrombosis, and heparin activation. Blood 1998, 92:2696-2706

27. Carrell RW, Lomas DA: Mechanisms of disease - Alpha(I)-antitrypsin deficiency - A model for conformational diseases. $N$ Engl J Med 2002, 346:45-53

\section{Pre-publication history}

The pre-publication history for this paper can be accessed here:

http://www.biomedcentral.com/1471-2466/2/5/prepub

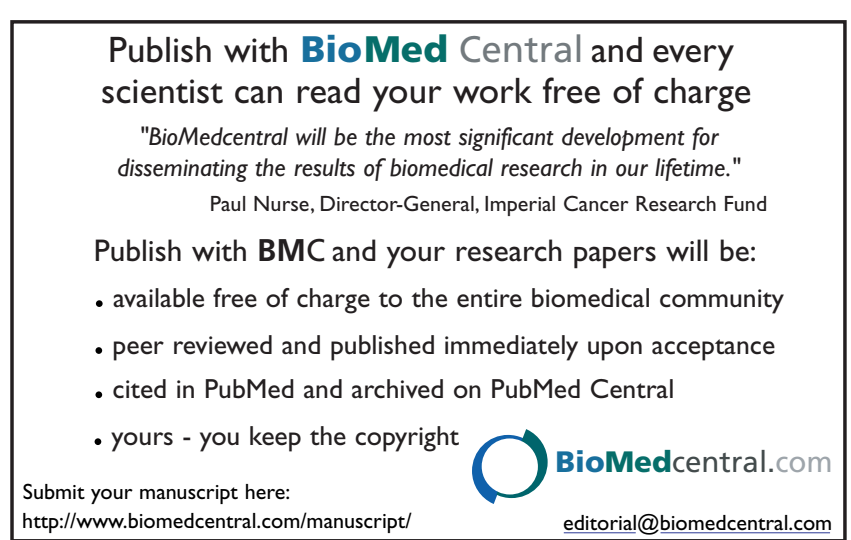

